\title{
Conventional Hydraulic Circuits in an Analysis of Environmental Issues
}

\author{
Boris M. Kaganovich ${ }^{1}$, Valery A. Stennikov ${ }^{1}$, Maxim S. Zarodnyuk ${ }^{1}$, Sergey V. Yakshin ${ }^{1}$ \\ ${ }^{1}$ Melentiev Energy Systems Institute of Siberian Branch of the Russian Academy of Sciences, Lermontov str. 130, Irkutsk, Russia
}

\begin{abstract}
The paper addresses the problems of conventional circuit modeling in an analysis of anthropogenic pollution of nature. A joint use of two types of models is proposed: 1) a model of conventional hydraulic circuits and 2) a model of extreme intermediate states developed by Melentiev Energy Systems Institute. The first model is used to calculate the distribution of pollutants in the atmosphere and their precipitation. The second model is intended for an analysis of the formation of pollutants and their transformations in branches as a result of chemical reactions and phase transitions. The analysis is based on the propositions of classical equilibrium thermodynamics. The applicability of the thermodynamic equilibrium and extremality principles and one-dimensional circuit models in the study of both reversible and irreversible processes are substantiated. Specific problems are analyzed. These are the determination of the yield of harmful substances in fuel combustion and processing, the distribution of pollutants in the atmosphere of large regions, the impact of hydro and wind power plants on the formation of harmful solutions in the air. The possibility of using a conventional graph (a thermodynamic tree) proposed by A.N. Gorban to analyze fuel technologies is demonstrated. The statement of the problem related to the assessment of the environmental compatibility of integrated energy systems is presented. The admissibility of the representation of the optimal distribution of flows in the integrated system as a sum of optimal distributions in its subsystems that differ from one another in physical-technical and economic nature is explained.
\end{abstract}

\section{Introduction}

The idea of modeling the conventional hydraulic circuits belongs to Kirchhoff. In [1], he solved the problem of distributing the electric current in a flat circular plate, by representing the plate as a graphcircuit, and the current - as a parallel motion of two positively and negatively charged liquids in equilibrium. The assumed statement of the problem revealed the most important advantage of one-dimensional space of variables, i.e. the mandatory potentiality of the continuous medium motion that occurs in it. Kirchhoff proposed two methods to search for the distribution of current. One of them [1] was based on solving a closed system of algebraic equations that describe the mass and energy balances in the circuit that satisfy the first and second laws further named after Kirchhoff. The applicability of another method follows from the heat theorem devised in [2]. According to this theorem, the isothermal current distribution in passive (without electromotive force sources) circuit corresponds to the minimum possible heat release. Since in isothermal processes heat is uniquely associated with entropy, apart from the mentioned heat minimum, there is also minimum production of entropy. Therefore, the above theorem can be regarded as a special case of the Onsager-Prigogine theorem, which is one of the basic theorems of non-equilibrium thermodynamics that was devised one hundred years later. Thus, Kirchhoff demonstrated the possibilities of the effective use of the mechanical-thermodynamic extremality principles by using conventional circuit modeling. It is worth noting, that he found the regularity considered to refer to the non-equilibrium theory, based on a purely equilibrium analysis.

The idea of Kirchhoff was further developed by Maxwell [3]. According to his heat theorem, the minimum transformation of an organized motion into a chaotic thermal one that was established by Kirchhoff, occurs when a linear relationship between the loss of voltage and current, i.e. Ohm's law, is maintained in the electrical circuit. Maxwell's theorem, as well as the theorem proved by Kirchhoff, can be regarded as a special case of the Onsager-Prigogine theorem. An analysis of the irreversible current distribution in [3], similar to that described in $[1,2]$, was based on the assumptions that the principles of equilibrium, potentiality and extremality are observed. In [3], using the method of potentials Maxwell supplemented the loop current method presented in [1] by demonstrating the possibility of calculating current distribution, and thus considerably deepened the analysis of the interrelationships between the theory of circuits and the equilibrium thermodynamics. He made an invaluable

Corresponding author: max@isem.irk.ru 
contribution to the development of macroscopic thermodynamics and statistical mechanics.

However, whereas the loop currents and nodal methods proposed by Kirchhoff and Maxwell were widely used in the analysis of electrical and hydraulic network operation, their thermal theorems faded into oblivion in both electrophysics and thermodynamics. The thermodynamic-circuit ideas began to gradually penetrate into the physical-mathematical and technicaleconomic modeling only in the second half of the twentieth century. The founder of the theory of hydraulic circuits, V.Ya. Khasilev, in contrast to Kirchhoff and Maxwell, included not only the distribution of flows in the object of his research, but also the optimal synthesis (selection of schemes and parameters) of modeled networks [4]. Optimizing rail and road transport routes, Kantorovich developed the ideas close to Khasilev's ideas [5]. If in a general case the costs of goods transportation between two specified nodes are determined by a selected route, the obtained optimal transportation scheme proves potential. The properties of potentials are characteristic of the prices for delivery of a cargo mass unit from any node to all the other nodes under an assumed price. The cost of transporting this mass unit between the nodes is determined by the price difference. In his models, Kantorovich presented the considered discrete transport flows by continuous conventional flows. Such an interpretation, without changing the properties of the applied models, can be replaced by the idea of motion of a liquid continuous medium, i.e. we can switch to the terms of the theory of hydraulic circuits. Further generalizations enable thermodynamic-circuit description of the other diverse macroscopic processes.

The use of the legacy of classics in conventional onedimensional circuit modeling for the analysis of environmental issues of the economic and energy development is the object of this research.

\section{Construction and use of conventional circuit models in environmental studies}

The laboratory of thermodynamics at Melentiev Energy Systems Institute started the research into the environmental issues related to the energy development with the construction of two fundamentally new thermodynamic models [6-10]. The first of them, the model of extreme intermediate states (MEIS), is designed to search of a state corresponding to the extreme value of a modeled system property of interest to the researcher (for example, the maximum content of harmful substances) in a multidimensional continuous space on a set of thermodynamic attainability. The second model aims to find the distribution of any real or conventional flow in the circuit from the condition of extremality of characteristic thermodynamic function. The need for simultaneous joint application of both models in solving individual problems was identified. For example, the distribution of polluted air flows in the analysis of the pollutants distribution in the atmosphere is calculated using the circuit model, while MEIS is used to assess the changes in the content of pollutants in the branches of a selected circuit due to chemical reactions or phase transitions. The jointly developed models have also been used in the optimal synthesis of physicalchemical and technical-economic systems. At the same time, it appeared to be possible to take into account the environmental requirements both by including special constraints in mathematical statements of the problems, and by selecting the "environmental" objective functions.

A comprehensive physical-mathematical analysis of the solved problems was necessary to find out the areas of effective application of the models [10]. The proof of the equilibrium thermodynamics applicability in modeling of irreversible trajectories was the key point in this analysis. Thus, two methods found their use: a stepby-step method and a circuit method [10]. The first method is intended for research in a multidimensional space of variables. The steps are taken as finite, but so small that the assumption of motion stationarity and, consequently, the invariability of its regularities, becomes admissible. This assumption made it possible to establish mathematical relationships between the theories of conservative and dissipative systems. The authors of [10] started to employ the method of dynamic programming to buildup the extreme results of the calculations during transition from one step to the next. The use of the second circuit method demonstrated that it was unnecessary to split the computational process into steps due to the potentiality inherent in onedimensional space in the presence of both conservative and dissipative motions in it.

In connection with the extreme nature of the investigated circuit modeling, we made an in-depth analysis of some heat theorems that were initial in the development of the theory of circuits and that determine the criteria and conditions for optimality of flow distribution. First of all, unlike Kirchhoff and Maxwell, we managed to devise these theorems not from the experimentally established Joule law, but from the principle of virtual displacements of Lagrange (equilibrium equations of a mechanical system) and the second law of thermodynamics [10]. Such conclusions helped to reconcile the discussed theorems with the propositions of classical mechanics; and equilibrium, nonequilibrium, and relativistic Planck-Einstein thermodynamics. The "universality" of the heat theorems became even clearer after they were extended to active circuits. Based on the method of Lagrange multipliers, an analysis of the flow distribution in such circuits indicated the maximum possible work of driving forces in them and the minimum production of heat and entropy that are not associated with this work. Thus, the principle of frugality, economical expenditure of energy resources, is also observed in the active circuits. The circuit processes, with the exception of the superfluidity case, are irreversible, therefore, the extensibility of equilibrium modeling to irreversibility phenomena in any macroscopic systems becomes also understandable.

The above interpretations of heat theorems demonstrate also the convenience of thermodynamiccircuit modeling of irreversible anthropogenic pollution of nature. To date, the objects of the environmental 
studies at Melentiev Energy Systems Institute has included: the formation of pollutants in fuel combustion and processing, the spread and transformation of pollutants in the atmosphere, the environmental compatibility of integrated energy systems (IES), and the impact of hydro and wind power plants on physicochemical processes in the atmosphere.

The study of the listed objects started with the investigation of the formation of harmful substances in fuel combustion and processing. The main tool in these studies was MEIS. Moreover, the application of its modification, including the circuit component $[6,7,10]$, also began. The development of this modification was based on the idea of A.N. Gorban, that was connected with the transformation of the set of thermodynamic attainability mapped on the polyhedron of mass balance, into a graph, i.e. a thermodynamic tree $[11,12]$ with potential thermodynamic functions monotonously changing along its branches. This property determines the fact that this tree has simultaneously the advantages of thermodynamic and one-dimensional circuit spaces of variables. The computational experiments show both a rather wide applicability of Gorban's idea and very high accuracy of the obtained results [13]. The construction of an algorithm that allows solving complex problems of large dimension with the help of a thermodynamic tree however still requires serious efforts.

An environmental analysis of fuel combustion and processing on the basis of conventional circuit modeling required the representation of mechanisms of the studied reactions in the form of circuits whose branches are individual stages of an overall process [6, 7, 10, 14].

With regard to the simplicity and efficiency of calculations, the use of the circuit modification of the model of extreme intermediate states in comparison with the multidimensional model proved to be much less successful. The initial information in the traditional MEIS is specified only by a list of modelled process components (for example, the reagents of chemical reactions) but not by a description of all its stages. This greatly simplifies the preparation of the initial data (information on the complete mechanism of complex processes usually cannot be obtained) and the computational experiment itself. Sometimes, however direct modeling of mechanisms helps to identify their dependence on the conditions of the studied process, the initial composition of reacting mixture, and the choice of catalysts. Therefore, further improvement in the circuit modification of MEIS is advisable.

The physical-chemical and technical-economic analyses of the applicability of conventional circuit models in the studies of anthropogenic pollution of nature make it possible to give a simple and concise mathematical formulation of the problems solved with the help of these models. The model of formation, transformation and distribution of pollutants in the atmosphere of large regions is the main in the discussed area of theoretical and applied research and has the form: find

$$
\min \sum_{\mathrm{i}=1}^{\mathrm{n}} z_{i} x_{\mathrm{a} i}^{\beta_{i}+1}
$$

(1)

(2)

$$
\text { subject to }
$$

$$
\mathbf{A} \mathbf{x}_{\mathrm{a}}=\mathbf{Q}_{\mathrm{a}},
$$

$$
\begin{gathered}
\sum_{j k} x_{\mathrm{d} k i}=0, \sum_{\ell k} x_{\mathrm{d} k i}=0, \\
x_{\mathrm{d} i}^{\mathrm{end}}=f\left(x_{\mathrm{d} i}^{\mathrm{beg}}\right)
\end{gathered}
$$

where $\mathbf{x}_{\mathbf{a}}$ - vector of air flows in the circuit branches; $z_{i}$ - resistance of the $i$-th branch; $\beta_{i}$ - exponent in the formula relating pressure loss in branch with flow in it; $\mathbf{A}=\left[a_{i j}\right]-(m-1) \times n-$ node-branch incidence matrix; $n$ - number of branches; $m$ - number of nodes; $\mathbf{Q}$ vector of external air sources and sinks; $j$ and $\ell-$ indices of nodes of air and pollutants sources and sinks, respectively; $\mathbf{x}_{\mathbf{d} k}$ - vector of flows of the $k$-th pollutant in the branches connecting at the $j$-th or at the $\ell$-th node; $\mathbf{x}_{\mathbf{d} i}^{\text {end }}$ and $\mathbf{x}_{\mathbf{d} i}^{\text {beg }}$ - vectors of pollutant flows at the final and initial points of the $i$-th branch, respectively.

Equation (2) represents a mass balance of air sources and sinks, and (3) is used to describe such balances for pollutants at the air and pollutants junction nodes.

Transition from $\mathbf{x}_{\mathbf{d} i}^{\text {beg }}$ to $\mathbf{x}_{\mathbf{d} i}^{\text {end }}$ as a result of chemical reactions and phase transitions, and inflow of pollutants due to anthropogenic emissions to the atmosphere are calculated using MEIS that with fixed temperature $T$, pressure $P$ and initial set of reagents $y$ has the form:

$$
\text { find } \quad \operatorname{extr}\left(\sum c_{\mathrm{dj}} x_{\mathrm{d} j}\right)
$$

$$
\begin{gathered}
D_{\mathrm{t}}(\mathbf{y})=\left\{\mathbf{x}: \mid \begin{array}{c}
\mathbf{x} \leq \mathbf{y}, \\
\varphi_{r}(\mathbf{x}) \leq \psi_{r}, r \in R^{\lim },
\end{array}\right\} \\
G(\mathbf{x})=\sum_{j} G_{j}(\mathbf{x}) x_{j}, \\
x_{j} \geq 0
\end{gathered}
$$

where $c_{\mathrm{d} j}$ - coefficient ranking hazard of the $j$-th pollutant; $\mathbf{A}-(m \times n)$ - matrix of element content in reagents; $\mathbf{x}=\left(x_{1}, \ldots, x_{n}\right)^{T}$ - vector of composition (mole quantities of reagents); $b$ - vector of mole quantities of elements; $\mathbf{y} \subset \mathbf{x}-$ vector of initial composition; $D_{\mathrm{t}}(\mathbf{y})$ - area (set) of thermodynamic attainability from the initial state; $\varphi_{r}$ and $\psi_{r}$ - limiting kinetic function of the $r$-th component $\mathbf{x}$ and its limiting value; $R^{\lim }-$ a 
set of indices of constraints on macroscopic kinetics; $G$ - Gibbs free energy.

The simplest scheme of the conventional hydraulic circuit, which serves for the graphic interpretation of models (1)-(4) and (5)-(10) is shown in Fig. 1. The nodes of the pollutant sources in this scheme correspond to locations of emission sources (energy, industrial, household). The location of hydro (HPP) and wind (WPP) power plants is also marked. The latter do not directly pollute the atmosphere, but affect air pressure and humidity, and, consequently, the formation of harmful solutions on the surface of water droplets.

When constructing the model (1)-(4), we assumed that the pollutant flows do not influence the distribution of air masses that are immeasurably larger in mass. Therefore, they were not included in the formula of the objective function (1).

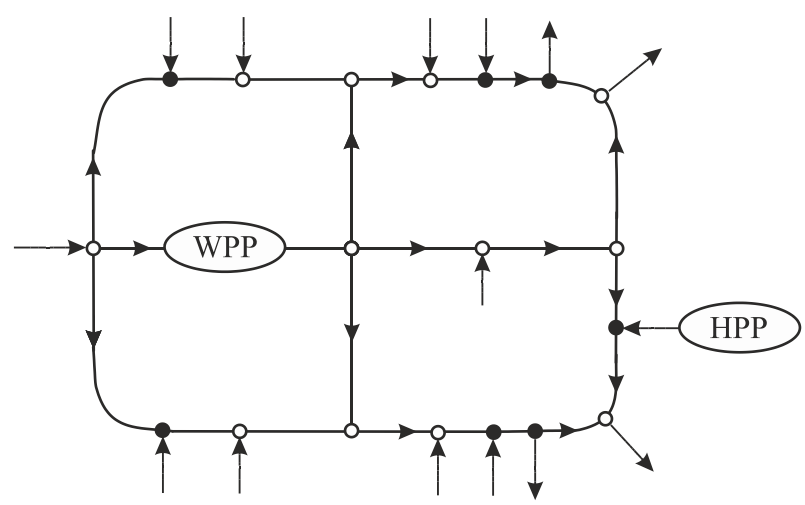

Fig. 1. Conventional hydraulic circuit. $\bigcirc-$ connection node of "air" branches; • - node of pollutant source (sink).

The model (1)-(4), including the block (5)-(10), can calculate the formation, transformation, distribution in large regions and falling with precipitation of a sufficiently large number of pollutants entering the atmosphere. This model can also be effectively used in solving the problems of optimal synthesis. To simplify the explanation, this problem can have the following formulation for the environmental analysis:

find

$$
\min \sum \mathbf{c}_{\mathbf{d} k} \mathbf{x}_{\mathbf{d} k}\left(\mathbf{x}_{\mathbf{a}}(\mathbf{z})\right)
$$

where $\mathbf{z}$ - the vector of resistance of branches that belong to the given redundant circuit scheme. The objective function (11) can be replaced by another function that means economic (monetary) costs. In this case, the environmental aspect of the considered issue should be included in the conditions for the maximum permissible pollution of nature in the system of the model constraints. The implementation of each of the two noted synthesis options is associated with considerable difficulties. Melentiev Energy Systems Institute started to successfully use the method of multiloop optimization that was proposed by S.V. Sumarokov to overcome these difficulties [15].

According to this method, the process of optimal choice of circuit schemes and parameters is divided into two stages. The first stage suggests calculation of flow distribution with fixed components of vector $\mathbf{z}$ on a given redundant scheme. In the second stage, the closed redundant scheme is transformed into a tree. At the end nodes of this tree, the conventional sources and sinks are specified to maintain invariable flows in the branches. Then, on the obtained tree, we select the resistance $\left(z_{i}\right)$ that corresponds to the optimal value of the chosen objective function. The choice of $z_{i}$ is made by the method of dynamic programming (DP). The indicated stages are iteratively repeated. The circuit branches where the flow rates reach the specified negligible values are removed from the circuit. The computational process is completed when the difference between the objective function values at two successive iterations becomes less than a given insignificant value. The multiloop optimization method has so far been sufficiently fully tested in terms of feasibility and usefulness only in the optimization of pipeline networks. There are plans to apply the method in modeling of transport integrated energy systems. The models of IES include the descriptions of both real electrical and hydraulic networks, and conventional ones that demonstrate the routes of discrete rail and road traffic. The convergence of multiloop optimization method is rigorously justified only for the hydrodynamic interaction between flow rates in the branches within the circuit, and the circuit as a whole has unique thermal interaction with the environment.

The use of conventional models to deal with the environmental aspect in the analysis of optimal synthesis problems involves solving many complex problems. One of the most important and difficult to solve among them is the problem of implementing the dynamic programming method for modeling the systems with non-economic objective functions. Thus, with the minimization of the atmospheric pollution at individual nodes of the territorial region, the choice of the conventional circuit scheme that should truthfully reflect a step-by-step process of pollution accumulation in the air, is non-trivial. It is clear that here the case in point is not the distribution of air flows in the atmosphere, but the conventional transport routes, for example, rail or road ones. It is necessary to use the research by Kantorovich mentioned in the introduction, and follow the principles of the discussed circuit modeling for the integrity of the studies and their presentation.

\section{An analysis of specific environmental issues}

Even solving the first of the problems considered in the paper, which is related to the search for concentrations of useful or harmful by-products obtained in the fuel combustion and processing, demonstrated strong possibilities of equilibrium thermodynamic modeling in the analysis of physicochemical, technical, economic and environmental problems. First of all, the comparative simplicity of carrying out complex computational experiments was discovered. The traditional theoretical kinetic analysis of the formation of even one hazardous pollutant (nitrogen oxide, hydrocyanic acid, 
formaldehyde, benzopyrene and many others) usually requires field experiments in addition to numerous calculations. The scope of work dramatically increases mainly due to the preparation of information on the mechanism of the modelled process. An extreme equilibrium analysis, that needs specification of a list of substances and information about only a few reactions limiting the speed of the process, immeasurably reduces the amount of initial information. There is often in principle unavailable information about the mechanism because the object of research is insufficiently studied. Accordingly, the stated problem becomes unsolvable. The unsolvability can sometimes be replaced by low quality and low applicability of the obtained results. Consequently, the transition from kinetic to thermodynamic modeling in a number of cases can ease the research done and increase its significance.

A further increase in the efficiency of equilibrium modeling using MEIS in at least some respects should be achieved by including the conventional circuit component in the applied models. The results of the application of modified MEIS indicate that the Gorban tree makes it possible to estimate the limiting (including environmental) levels of perfection and degradation of natural and techno-economic systems for the simplest examples [13]. It has become possible to comprehensively analyze the course of various reactions depending on their conditions: fuel preparation and its mixing with air, hydrodynamics, heat transfer, macrokinetics, diffusion, the phase boundary formation and many other factors.

The environmental conventional circuit modeling has so far found the greatest use in the analysis of atmospheric pollution $[6,7,10,16]$. The first original and important result of this analysis was the substantiation of the very possibility of making it. There was a widespread opinion about the absolute inapplicability of thermodynamics in the study of atmospheric chemical processes. The explanation of its validity was based on the idea of a relatively low air temperature, and hence the low rate of reactions that occur at such a temperature and the unattainability of the final equilibrium point $\boldsymbol{x}^{\mathbf{e q}}$. MEIS has eliminated the possibility of this explanation. This model in contrast to traditional equilibrium thermodynamic models, can be used to study along with $\boldsymbol{x}^{\mathbf{e q}}$ the entire region of $\boldsymbol{x}^{\mathbf{e q}}$ attainability, and to find partial equilibria points of interest for the researcher involved in the computational experiment. The accuracy and rich content of the thermodynamic studies of chemical transformations in the atmosphere have been repeatedly confirmed.

The high quality of the results was obtained under the idealization of the problem associated with overcoming many difficulties. The difficulties are determined by the complexity of the modeled object. The atmosphere represents an open heterogeneous system interacting with a heterogeneous environment. It includes a gas phase, water droplets and aerosols (solid and liquid, of anthropogenic and natural origin). Condensed particles generally have a spatially inhomogeneous structure, including solutions on the surface. The matter of the atmosphere is unevenly distributed vertically (due to a decrease in the air density with the height) and horizontally due to the presence of air flows. Changes in the composition and spatial distribution of matter occur under the action of a variety of forces: gravity, wind pressure, chemical affinity, surface tension, electrical interactions, etc. The processes of energy, matter, and charge transfer caused by these forces often differ in essential irreversibility, and in some cases have a nonstationary character. Although one-dimensional thermodynamic-circuit modeling, in comparison with the models of multidimensional motion of a continuous medium, greatly facilitated the overcoming of the above difficulties, even simplified problems proved to be difficult to accomplish.

The solved problems of the atmospheric pollution analysis can be divided into two groups. The first represents a static study of the pollution of a given volume of a continuous medium. The second is related to modeling the motion of air-and-pollutants flows. From the first group it is necessary to identify the problems of: the phase equilibrium of polluted mixtures, the formation and transformation of pollutants in certain phases, and the formation of harmful solutions on the surface of condensate droplets. In the second group, the most interesting were: 1) assessment of the impact of hydro and wind power plants on physicochemical processes in the air and 2) modeling of formations, spreads, transformations and precipitation of pollutants in large regions.

Firstly, we will briefly note the results obtained when solving the problems of the first group. In the analysis of phase equilibria, one of the important results was the formulation of the model and the determination, with its help, of the limiting concentrations of vapor and condensed water at given air pressure and temperature. The achievement of this result is related to solving the computational problems arising when switching from studies of two- and three-dimensional systems (usually discussed in the literature) to studies of largedimensional systems. Moreover, it is difficult to use even known formulas for changing the potentials and pressures over a curved phase boundary in the modeling of multicomponent systems. Formula [10] was derived and used to change the Gibbs energy

$$
G_{\mathrm{g}}-G_{\mathrm{c}}=3 \alpha V_{\mathrm{c}} / r,
$$

where $G_{\mathrm{g}}$ and $G_{\mathrm{c}}$ - molar Gibbs energies of gas and condensed water phases, respectively; $\alpha$ - surface tension; $V_{c}-$ molar volume of condensed phase, $r$ droplet radius. Equation (12) differs from the conventionally used formula by a numerical factor (3 instead of 2). A natural explanation for the increase in the difference between the values of the thermodynamic potentials is the work performed to move one mole of water vapor from the space in which it is a gas mixture component to a one-dimensional space immediately adjacent to the drop surface. This problem became an obligatory part of the other two problems of the above first group. The results of the pollution analysis in cases where they could be compared with the literature and 
experimental data showed high accuracy of the calculations (errors did not exceed two to three percent).

The problems of the HPP and WPP impact assessment that belong to the second group of problems were solved using the conventional hydraulic circuits (Fig.1). Unlike thermal power plants that directly pollute the air, hydroelectric power plants and wind turbines affect physical-chemical processes in the air and, in particular, the formation of harmful solutions on the surface of water droplets.

Hydropower plants as well as wind power plants have been considered environmentally absolutely clean for many years. However, with an increase in the unit capacities, the number of the plants and their share in the energy balances of countries, the negative impact of hydropower on nature has become increasingly serious. The danger of flooding large areas, the impact on the viability and reproduction of the aquatic environment inhabitants and on coastal vegetation have been identified. The increase in air humidity in the areas of reservoirs has led to a change in the rain conditions and an increase in the pollution of precipitation. Indeed, the rise in the number of drops and increase in their surface in the atmosphere lead to an increase in the formation of harmful solutions and, eventually, to the "dirt" falling on the earth's surface.

Wind energy is developing rapidly, the unit capacities of wind turbines are increasing to hundreds of thousands and millions of kilowatts, therefore, it is necessary to assess the probability of environmental problems similar to those accompanying operation of hydropower plants. The volumetric energy intensity of the air (which determines the speed-dependent energy of motion) is approximately 750 times less than the energy intensity of the water. Therefore, in the case of close capacities of hydraulic and wind power plants, the latter consume immeasurably larger flows of continuous medium than the former. The use of wind farms to cover the peak part of the load curve can result in a rapid change in the atmospheric pressure in these flows. The change in pressure causes even a sharper change in humidity, as shown in Fig. 2 borrowed from [10].

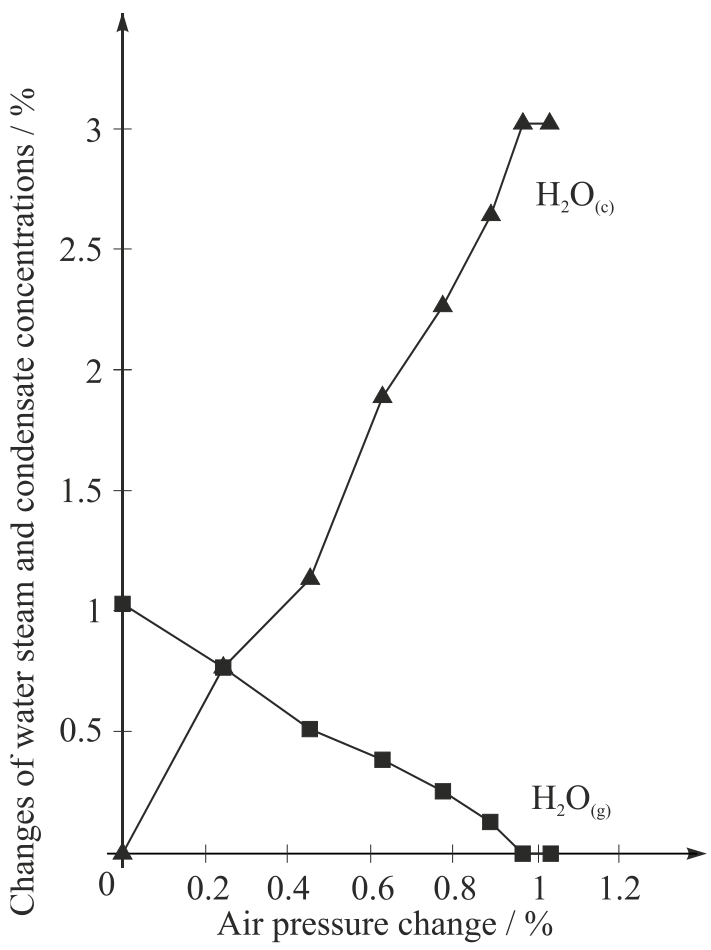

Fig. 2. Relative relationships between the concentrations of water vapor (g), condensate (c) and a change in the atmospheric pressure at $\mathrm{T}=293 \mathrm{~K}$.

Indeed, the pressure drop due to energy use for driving wind turbines also leads to a decrease in condensed water concentration and it seems that under the influence of wind power plants the hazardous rain precipitation should decrease. However, its decrease close to power plants can give rise to more intensive "hazardous" rains on more distant sections of air motion. The probability of such a run of events increases with construction of several large wind power plants on a relatively small area. Therefore, a detailed analysis of environmental compatibility of wind power plants must intensively be expanded in terms of socioeconomic and climatic conditions at the sites of their construction, surface relief and some other factors. It must also include an assessment of the direct impact of fluctuations in wind conditions on a human being.

In fact, the studies of pollutants distribution and transformations in the atmosphere and their precipitation in large regions considerably expand an application sphere of the conventional modeling in comparison with the considered modeling of indirect environment pollutions. The latter is included in the distribution analysis as a component part. Meanwhile, besides the analysis of environmental compatibility of hydro and wind power plants model (1)-(4) for pollution distribution is applied to the below study of ecological characteristics of integrated energy systems. However, even the first steps in application of conventional hydraulic circuits in this direction facilitated understanding of its high applied and theoretical relevance.

The applied relevance stems primarily from the possibility of a detailed and multilateral analysis of air pollution in large regions. It can comprise determination 
of the role of individual industrial enterprises, power plants, transport, public utilities in degradation of environmental situation; the pollution levels in individual areas; weather stability depending on the atmospheric pressure fluctuations, air humidity and flows of pollutants along the branches of the applied conventional circuit. The obtained information proves to be highly useful for addressing the environmental issues in development of the studied region, in particular its energy sector. Consideration of the environmental standards when choosing the updated structure of the regional energy sector and searching for the optimal conditions of using energy carriers is necessary and possible for tackling the energy issues.

In the theoretical issues of modeling the distribution of pollutants, which are linked with the accuracy of applied issues, choice of the schemes of conventional circuits is apparently the most important and difficult issue. The optimality criterion, i.e. the maximum accuracy of determination of the real air distribution in the modeled region, is clear to a considerable extent in its analysis. But the measurements of this accuracy are also an intractable challenge. Probably, it can be estimated using the final results of calculations, i.e. concentrations of pollutants at different points of the chosen scheme because their measurement does not cause essential difficulties. Identification of the level of their detail will certainly become a complex component to be determined. It will be necessary to address many issues to provide an insight into physical and mathematical features of the computational methods on the specified schemes.

Let us discuss the search for distribution of energy carriers flows in the transport integrated energy systems and the optimal synthesis of these systems that is the last analyzed problems of conventional circuit modeling. At Melentiev Energy Systems Institute, development of IES is treated as a promising trend in energy [17]. From the standpoint of the thermodynamic-circuit analysis, the models of energy integration are interesting as descriptions of objects containing different physical phenomena. Such phenomena can be studied jointly using the general principles of conservation, equilibrium, potentiality and extremality, and the one-dimensional space of variables, in which these principles are necessarily observed.

The capabilities of the conventional circuit modeling of integrated energy systems will be illustrated by the example of a transport energy system consisting of three types of energy transport: electric and pipeline heat networks and railway transport of fuel. A simple scheme of such an energy system is presented in Fig. 3.

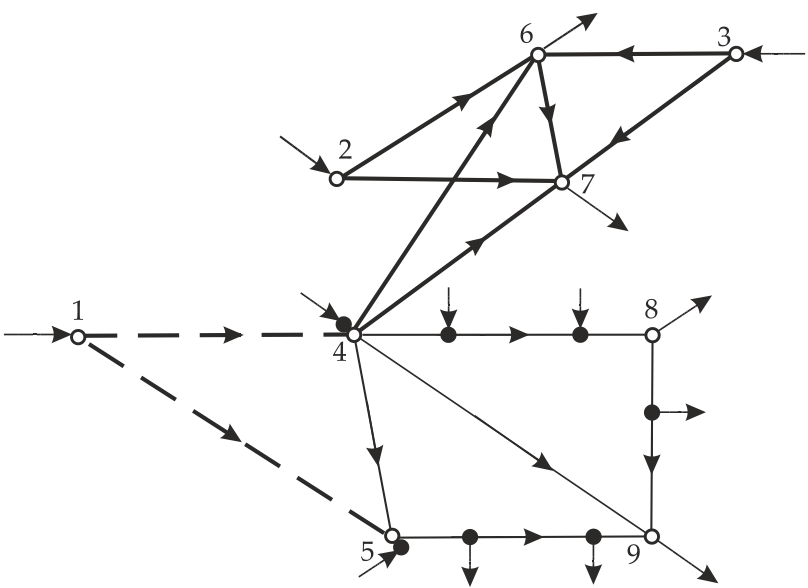

Fig. 3. A conventional circuit of an integrated energy system. Nodes: 1 - gas source; 2 - coal source by railway, thermal power plant (TPP); 3 - electricity source (HPP); 4 cogeneration plant; 5 - boiler plant; $\bigcirc$ - source (sink) of energy carriers; - - source (sink) of pollutants. Branches: 1-4, 1-5 - gas pipelines; 2-6, 2-7, 3-6, 3-7, 4-6, 4-7, 6-7electric networks; 4-5, 4-8, 4-9, 5-9, 8-9 - heat networks.

The model of energy carrier flows distribution has the following form:

find

$$
\min \sum_{i=1}^{n} \mathrm{z}_{\mathrm{si}} x_{\mathrm{si}}^{\beta_{\mathrm{si}}+1}
$$

$$
\text { subject to } \quad \mathbf{A}_{\mathrm{s}} \mathbf{x}_{\mathrm{s}}=\mathbf{Q}_{\mathrm{s}} \text {, }
$$

where $s$ - an energy carrier index. The matrix equations of material balances (14) are written for each subsystem corresponding to the used energy carriers. In order to determine flow distribution, branches $1-4$ and 1-5 are removed from the calculation scheme, and the sources corresponding to the flows in these branches are assigned at nodes 4 and 5 .

The minimum of objective function (13) as a whole and its parts relating to individual subsystems is observed owing to inactivity of the chosen schemes. From minimization of energy consumption for transportation of energy carriers we can pass to minimization of cash expenditures and then to solve the optimal synthesis problem, i.e. to choose schemes and parameters of a transport energy system. A redundant scheme is required for the analysis of the last indicated problem. As a result of optimization the excess branches, which make construction and operation of the transport energy system more expensive, must be eliminated.

The environmental standards are taken into consideration in the computational experiments by addition of the source and sinks of pollutants to the scheme in Fig. 3. As a result, it becomes possible to supplement model (13), (14) with material balances of pollutant flows (3) and equation of pollutant transformations (4). The environmental analysis can certainly be carried out using the optimal synthesis model with the minimized objective function implying a concentration of the mixture of pollutants in the atmosphere of the considered region.

The suggested methods of representing the result of system optimization as a sum of optimal modeling 
results of different physical and technical-economic components are admissible owing to generality of the applied regularities and their obligatory observation in the one-dimensional space of variables.

\section{Conclusions}

The studies of fruitfulness of the conventional circuit modeling for the analysis of environmental issues are in the initial stage of development. We analyzed only several specific problems. A set of numerous issues to be solved has not been determined yet. However, capabilities and need to expand an application area of conventional hydraulic circuits became clear.

The capabilities are determined primarily by the explained comparative simplicity of the one-dimensional models which is conditioned by the mandatory potentiality of the one-dimensional space of variables. Simplicity consists in the admissible application of universal principles of equilibrium and extremality to modeling of both reversible and irreversible processes; in description of these two opposite motion patterns by the language of mathematical programming.

The advantages of the one-dimensional space rise even more owing to the convenient solution of problems in the thermodynamic space possessing monotone characteristic functions. This additional utility turned out particularly useful for description of chemical transformations and phase transitions of pollutants on individual branches by equality (4). After all, for searching for the changes in the values of variables, which occur in these processes, we had to switch from the one-dimensional to multi-dimensional spaces.

Applicability of the one-dimensional modeling was illustrated initially by the examples of fuel processing and combustion. Transformation of a set of thermodynamic attainability into a circuitthermodynamic tree in the model of extreme intermediate states applied to this analysis made it possible to improve the accuracy of calculations and contributed to identification of physical and mathematical features of the solved environmental problems of pollutant formation.

The described examples of construction and application of conventional hydraulic circuits to the analysis of atmospheric pollution in large regions can and must serve as a starting point for development of a new section in the theory of hydraulic circuits conventional circuit modeling. It should include issues of the optimal choice of a conventional scheme, joint thermodynamic-circuit modeling, identification of physical and mathematical features of the problems of flow distribution and optimal synthesis on the conventional schemes. This statement of the problem on environmental compatibility of wind energy can suggest similar statements of environmental problems in utilization of renewable energy resources.

Substantiation of the solvability of optimization problems of flow distribution and choice of the scheme and parameters for the systems comprising different physical and technical-economic subsystems appears to be highly interesting in the stated issue on environmental effectiveness of integrated energy systems. Further studies of this issue must be a mandatory component part of the analysis of integration effectiveness in the energy sector.

\section{References}

1. G.R. Kirchhoff, Annalen der Physik und Chemie, 64, 497-514 (1845)

2. G.R. Kirchhoff, Annalen der Physik und Chemie, 75, 189-205 (1848)

3. J.C.A. Maxwell, Treatise on Electricity and Magnetism (The Clarendon Press, London, 1891)

4. Viktor Yakovlevich Khasilev: Memories of Life and Work. Scientific Legacy (Academic Publishing House "Geo", Novosibirsk, 2012)

5. L.V. Kantorovich, Reports of AS SSSR, 37, 227-229 (1942)

6. A.N. Gorban, B.M. Kaganovich, S.P. Filippov, Thermodynamic equilibria and extrema: Analysis of attainability regions and partial equilibria in physicochemical and technical systems (Nauka, Novosibirsk, 2001)

7. A.N. Gorban, B.M. Kaganovich, S.P. Filippov, A.V. Keiko, V.A. Shamansky, I.A. Shirkalin, Thermodynamic Equilibria and Extrema. Analysis of Attainability Regions and Partial Equilibria (Springer, NY, 2006)

8. B.M. Kaganovich, A.V. Keiko, V.A. Shamansky, Advances in Chemical Engineering. Thermodynamics and kinetics of complex systems, 39, 1-74 (Elsevier, 2010)

9. B.M. Kaganovich, A.V. Keiko, V.A. Shamansky, M.S. Zarodnyuk, Chemical Kinetics, 31-60 (Intech, Rijeka, 2012)

10. B.M. Kaganovich, Equilibrium thermodynamics. Problems and perspectives (LAP LAMBERT Academic Publishing, Saarbruecken, 2015)

11. A.N. Gorban, Equilibrium encircling: Equations of chemical kinetics and their thermodynamic analysis (Nauka, Novosibirsk, 1984)

12. A.N. Gorban, SIAM J. Applied Dynamical Systems, 12, 246-278 (2013)

13. M.S. Zarodnyuk, Application of the graph "thermodynamic tree" to equilibrium modeling of physicochemical systems (ESI SB RAS, Irkutsk, 2013)

14. B.M. Kaganovich, S.P. Filippov, Equilibrium thermodynamics and mathematical programming (Nauka, Novosibirsk, 1995)

15. S.V. Sumarokov, Mathematical modeling of water supply systems, (Nauka, Novosibirsk, 1983)

16. B.M. Kaganovich, A.P. Merenkov, O.A. Balyshev, Elements of heterogeneous hydraulic circuits theory (Nauka, Novosibirsk, 1997) 
17. N.I. Voropai, V.A. Stennikov, Proceedings of the Russian Academy of Sciences. Energetika, 1, 64-72 (2014) 